\title{
Identification of train loads from the dynamic responses of an integrated sleeper in-situ
}

\author{
Le-Hung Tran ${ }^{1,2}$, Tien Hoang ${ }^{1}$, Gilles Foret ${ }^{1}$, Denis Duhamel ${ }^{1}$, Samir Messad ${ }^{2}$ and Arnaud \\ Loaëc ${ }^{2}$
}

\begin{abstract}
The monitoring of railway tracks can be performed through several measurement techniques. Recently, a method of diagnosing the railway track has been proposed using Fiber Bragg Gratings (FBG) integrated inside the railway sleeper. An analytical model for the dynamics of railway sleepers has been developed allowing calculation of the sleeper responses. In this model, by using the relation between the rail forces and displacements of a periodically supported beam, the sleeper's dynamic equation is written with the help of the Euler-Bernoulli beam and Dirac's delta distribution. Subsequently, the sleeper dynamic responses are calculated using the Green's function. This article presents an application of this model to identify the train loads from the strains measured in-situ. Based on this model, we can obtain a matrix which presents the link between the loads and the sleeper responses. Then, by substituting the Fourier transform of measured strains at the middle and at the two rail-seats of the sleeper, the train loads can be quickly calculated by inverting the matrix with the help of MATLAB. This method is validated by the experiments. Numerical examples with the measurement in-situ are presented to show identified wheel loads from experimental signals.
\end{abstract}

\section{Keywords}

Railway Dynamics, Instrumented Sleeper, Fiber Bragg gratings, Green's function, Computation methods

\section{Introduction}

Generally, defective materials can be detected by several different methods. For example: image analysis, measurement of elastic waves, electrical resistance or acoustic emission focus on a variation of the propagation time of waves and electrical resistance to detect the internal cracks of objects or modal analysis. But in recent years, a novel technology using the Fiber Bragg Grating (FBG) to diagnose the railway track has been developed. Firstly, this kind of sensors was used on the rail or the rolling stock. Filograno et al. (2012) posed the FBG sensors on the rail in different directions (vertical, horizontal and inclined at an angle $45^{\circ}$ ) for monitoring the high speed line from Madrid to Barcelona. They could detect the train parameters: train speed and acceleration, the distance between the wheels and the total number of wheels. Moreover, by studying the electromagnetic interference (EMI) on the delicate changes of the FBG's measurements, Wei et al. (2010); Wei et al. (2012) proposed an approach of axle counting with two methods presented: $\mathrm{X}$-Crossing and $\mathrm{D}$ (erivative)-Crossing. In fact, a peak in the signal is determined by a rising edge, followed by a falling edge. X-Crossing method based on a definition of two different thresholds to detect the rising edge and the falling edge. However, the X-crossing method is not able to detect all peaks in some signals. Hence, D-Crossing method is developed to supplement the $\mathrm{X}$-crossing method by using the first derivative of the signal. In the program PMNIDEA* which is carried out under the European Commission's $7^{\text {th }}$ framework, Buggy et al. (2016) has used the FBG sensor to monitor the fish plate (joint of two rails). This work demonstrated a significant change in the high frequency component of the strain responses for different torque settings. Tam et al. (2007) presented the system "Smart Railways" which has been developed by KCRS's East Rail in Hong Kong. Two systems were installed on the rail tracks and on a board of train car to detect the wheel/rail response. The FBG sensor has been demonstrated to be able to detect the rail imperfection by Ho et al. (2006). Da Costa Marques Pimentel et al. (2008) developed a measurement system for the characterization of railway traffic and its application was realized on a short span railway bridge in Northern Portugal.

The FBG sensor was integrated also in the railway sleeper. Yazdizadeh et al. (2017) conclude that the FBG sensors cast inside the specimens worked properly. Therefore, the fiber-optic technique is a better alternative than electrical strain gauge for studying the time-dependent properties. In France, Loaëc and Petit (2014) presented a railway sleeper "Smart Sleeper" which has been developed by Sateba with 6 sensors allowing to measure the sleeper strain when the train is passing. Recently, Qian et al. (2019), researchers

${ }^{1}$ Laboratoire Navier, UMR 8205, École des Ponts ParisTech, Université Gustave Eiffel, CNRS, 77455 Champs-sur-Marne, Marne-la-Vallée Cedex 2, France

${ }^{2}$ Sateba, 33 places des Corolles, 92400 Courbevoie, France

\section{Corresponding author:}

Le-Hung Tran, Laboratoire Navier, UMR 8205, École des Ponts ParisTech, Université Gustave Eiffel, CNRS, 77455 Champs-sur-Marne, Marne-la-vallée Cedex 2, France

Email: le-hung.tran@enpc.fr

${ }^{*}$ Predictive maintenance employing non-intrusive inspection \& data analysis 
at the University of Illinois at Urbana-Champaign and the University of South Carolina presented a project "Railroad Infrastructure 4.0" which used an integrated railway sleeper to continuously quantify ballast pressure distribution under the sleeper. In this work, the strain gauges are fixed along the sleeper length with the same distance. The foundations are divided into six discrete bins of equal sizes and the ballast support condition is simulated via an assessment system. However, this work used an estimated reaction force which is calculated by an equation given by AREMA ${ }^{\dagger}$.

Substantial research using analytical and numerical methods for rail track has been carried out. Analytical models of the rail track have been developped by considering the model of an infinite beam placed on a continuous foundation by Ding et al. (2012); Nguyen and Duhamel (2006, 2008) or a periodically supported beam by Mead (1970, 1996); Sheng et al. (2005); Hoang et al. (2017). Some research focuses on the pre-stressed concrete sleeper using Finite Element Method (FEM) in 2D and in 3D (see Kumaran et al. (2003); Arab et al. (2011)). Tran et al. (2018) have developped an analytical model of the railway sleeper which allows us to calculate the sleeper response posed on a homogeneous foundation in a shorter time than numerical models.

In this paper, an "inverse problem" has been developed to determine the vertical train loads based on the analytical model of the railway sleeper which has been presented by Tran et al. (2018). By considering a beam resting on a Kelvin-Voigt foundation and by assuming a vertical periodic load, the sleeper strain can be calculated rapidly with the help of Green's function. The formulations of this model are recalled in the Section 2. So, a linear relation between the sleeper strain in the frequency domain and the vertical train loads is obtained with help of Green's function. Hence, the vertical train loads can be identified by using the MATLAB solver mldivide. We verify this "inverse problem" by back-calculating the train loads from a signal. That is a combination of random imposed loads and different levels of noise. Thereafter, the experimental validations are presented in the section 3. In this section, a sleeper is instrumented by the strain gauges at different zones. Two tests are already realized with an impact hammer and a passing train. In two cases, the loads applied on the sleeper are known. By using the method of identification of the train loads, the loads identified agree well with the applied loads. Another application has been shown in the Section 4 with the real measurements recorded in-situ by the "Smart Sleeper". The results of this application show the different train loads applied on each rail which corresponds to different strain level recorded by the FGB sensor of the sleeper. Finally, concluding remarks are drawn in Section 5.

\section{Formulations}

\subsection{Analytical model of the railways sleepers}

Let us consider that a railway track can be modelled as shown in the Fig. 1. In this dynamic model, the sleeper together with the ballast and foundation are modeled by an Euler-Bernoulli beam resting on a Kelvin-Voigt foundation. The Euler-Bernoulli beam model is good enough to describe a mechanical behavior of the railway sleeper for low
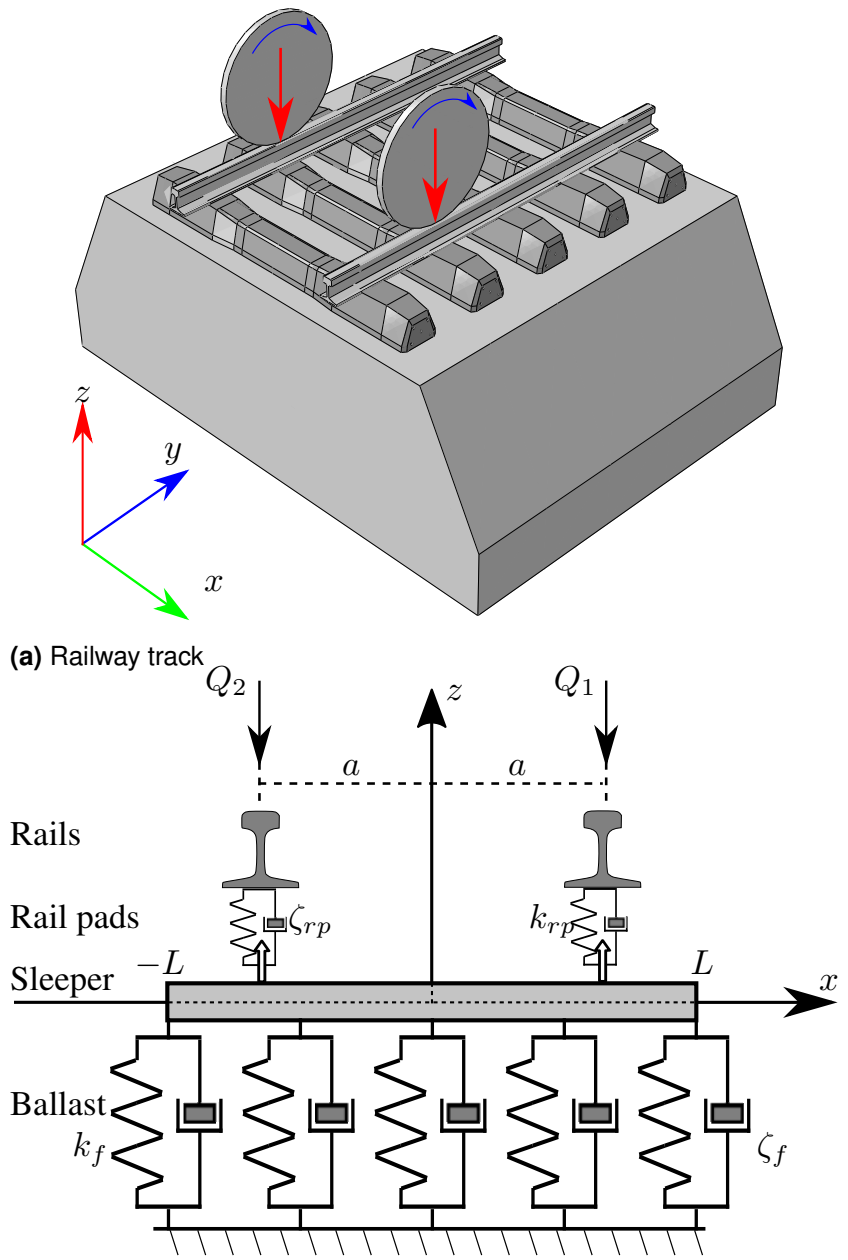

(b) Analytical model representation

Figure 1. Railway track (a) and the analytical model representation (b)

frequencies. The ballast layer features complex mechanical behavior and the Kelvin-Voigt foundation is a useful model for the viscous solid which takes into account an effect of damping's foundation.

In the Fig. $1 b$, the sleeper length is $2 L$ (from $-L$ to $L$ ) and the rail positions are at $x= \pm a$. The sleeper displacement $w_{s}(x, t)$ under a force $F(x, t)$ is driven by the dynamic equation of the Euler-Bernoulli pre-stressed beam and together the boundary conditions for a free-free beam as follows:

$$
\left\{\begin{array}{c}
E_{s} I_{s} \frac{\partial^{4} w_{s}(x, t)}{\partial x^{4}}+\rho_{s} S_{s} \frac{\partial^{2} w_{s}(x, t)}{\partial t^{2}} \\
-T \frac{\partial^{2} w_{s}(x, t)}{\partial x^{2}}+k_{f} w_{s}(x, t) \\
+\zeta_{f} \frac{\partial w_{s}(x, t)}{\partial t}=F(x, t) \\
\frac{\partial^{2} w_{s}}{\partial x^{2}}(-L, t)=\frac{\partial^{2} w_{s}}{\partial x^{2}}(L, t)=0 \\
\frac{\partial^{3} w_{s}}{\partial x^{3}}(-L, t)=\frac{\partial^{3} w_{s}}{\partial x^{3}}(L, t)=0
\end{array}\right.
$$

${ }^{\dagger}$ American Railway Engineering and Maintenance-of-Way Association 
where $\rho_{s}, E_{s}, S_{s}$ and $I_{s}$ are the density, the Young's modulus, the section and the cross-sectional moment inertia of the sleeper respectively and $T$ is the sleeper pre-stress. In this scenario, the sleeper is posed on a homogeneous foundation and the foundation's parameters are $k_{f}, \zeta_{f}$ which present the stiffness and damping coefficients respectively. We note that for a free-free beam posed on the foundation, the moment and the shear force are vanishing at two extremities, thus these boundary conditions can be imposed by the $2^{\text {nd }}$ and $3^{\text {rd }}$ partial derivative with regard to $x$ set to zero respectively and its expressions are detailled in the Eqs. (1b) and (1c).

By using the Dirac's delta distribution, the force applied by the rails on the sleeper $F(x, t)$ can be written as follows :

$$
F(x, t)=-R_{1}(t) \delta(x-a)-R_{2}(t) \delta(x+a)
$$

where $R_{1}$ and $R_{2}$ are the reaction forces applied on the rail positions $(x= \pm a)$ in time domain.

Eq. (1a) together with the boundary conditions (1b) and (1c) is a $4^{\text {th }}$ order linear differential equation in the frequency domain which can be solved with help of the Green's function $G(x, \omega)$ (see Appendix A). Hence, the sleeper response in the frequency domain $\hat{w}_{s}(x, \omega)$ can be rewritten as follow:

$$
\hat{w}_{s}(x, \omega)=\frac{-\hat{R}_{1}(\omega)}{E_{s} I_{s}} G_{a}(x, \omega)+\frac{-\hat{R}_{2}(\omega)}{E_{s} I_{s}} G_{-a}(x, \omega)
$$

where $\hat{R}_{1}(\omega)$ and $\hat{R}_{2}(\omega)$ are two reaction forces applied on the sleeper in the frequency domain.

Therefore, the sleeper displacement at two rail positions can be calculated by substituting $x= \pm a$ into the aforementioned equation:

$$
\begin{aligned}
& \hat{w}_{s}(a, \omega)=\frac{-\hat{R}_{1}}{E_{s} I_{s}} G_{a}(a, \omega)+\frac{-\hat{R}_{2}}{E_{s} I_{s}} G_{-a}(a, \omega) \\
& \hat{w}_{s}(-a, \omega)=\frac{-\hat{R}_{1}}{E_{s} I_{s}} G_{a}(-a, \omega)+\frac{-\hat{R}_{2}}{E_{s} I_{s}} G_{-a}(-a, \omega)
\end{aligned}
$$

By modelling a rail pad as a system of springer-damper, the reaction force can be calculated with the help of the model of the periodically supported beam in the frequency domain (see Appendix B) as follows:

$$
\left\{\begin{array}{l}
\hat{R}_{1}(\omega)=\frac{k_{p} \mathcal{K}}{k_{p}+\mathcal{K}} \hat{w}_{s}(a, \omega)+\frac{k_{p}}{k_{p}+\mathcal{K}} \mathcal{Q}_{1}(\omega) \\
\hat{R}_{2}(\omega)=\frac{k_{p} \mathcal{K}}{k_{p}+\mathcal{K}} \hat{w}_{s}(-a, \omega)+\frac{k_{p}}{k_{p}+\mathcal{K}} \mathcal{Q}_{2}(\omega)
\end{array}\right.
$$

By combining Eqs. (4) and (5), we obtain the result of the reaction force of the sleeper:

$$
\begin{aligned}
& \hat{R}_{1}=\frac{E_{s} I_{s}}{\tilde{D} \mathcal{K}}\left(\mathcal{Q}_{1}\left[G_{-a}(-a, \omega)+\chi\right]-\mathcal{Q}_{2} G_{-a}(a, \omega)\right) \\
& \hat{R}_{2}=\frac{E_{s} I_{s}}{\tilde{D} \mathcal{K}}\left(\mathcal{Q}_{2}\left[G_{a}(a, \omega)+\chi\right]-\mathcal{Q}_{1} G_{a}(-a, \omega)\right)
\end{aligned}
$$

where $\quad \chi=E_{s} I_{s} \frac{k_{p}+\mathcal{K}}{k_{p} \mathcal{K}} \quad$ and $\quad \tilde{D}(\omega)=$ $\left[G_{a}(a, \omega)+\chi\right]\left[G_{-a}(-a, \omega)+\chi\right]-G_{a}(-a, \omega) G_{-a}(a, \omega)$. The two coefficients $\mathcal{K}$ and $\mathcal{Q}_{k}$ are respectively an equivalent stiffness and equivalent charges which are defined in the Appendix B. The equivalent stiffness depends on railway track parameters and the equivalent load represents train loads in the frequency domain.

Then, the sleeper displacement in the frequency domain can be obtained by replacing $\hat{R}_{1}$ and $\hat{R}_{2}$ in Eq. (3). By using the beam theory, the sleeper strain in the longitudinal direction in the frequency domain for an Euler-Bernoulli beam $\hat{\varepsilon}_{x x}(x, z, \omega)$ can be calculated as follows:

$$
\begin{aligned}
& \hat{\varepsilon}_{x x}(x, z, \omega)= \\
& \frac{z_{s}}{E_{s} I_{s}}\left(\hat{R}_{1} \frac{\partial^{2} G_{a}}{\partial x^{2}}(x, \omega)+\hat{R}_{2} \frac{\partial^{2} G_{-a}}{\partial x^{2}}(x, \omega)\right)
\end{aligned}
$$

where $z_{s}$ is the distance to the beam's neutral axis. By using the inverse Fourier transform of Eqs. (3) and (7), we can get the sleeper response and the sleeper strain in the time domain.

\subsection{Method of identification}

In this part, we introduce the method of identifying the vertical train loads by using the dynamic model of the railways sleepers which we presented previously. By considering that the sleeper integrates several FBG sensors at different positions, the Eq. (7) can be rewritten as follows:

$$
\begin{aligned}
& \hat{\varepsilon}_{x x_{i}}\left(x_{i}, z_{s_{i}}, \omega\right)= \\
& \quad \mathbf{A}_{\mathbf{i} 1}\left(x_{i}, z_{s_{i}}, \omega\right) \hat{R}_{1}(\omega)+\mathbf{A}_{\mathbf{i} 2}\left(x_{i}, z_{s_{i}}, \omega\right) \hat{R}_{2}(\omega)
\end{aligned}
$$

where $\left(x_{i}, z_{s_{i}}\right)$ are the coordinate of the sensors $i$ and two parameters $\mathbf{A}_{\mathbf{i} 1}, \mathbf{A}_{\mathbf{i} 2}$ of the Eq. (8) are detailled as follows:

$$
\left\{\begin{array}{l}
\mathbf{A}_{\mathbf{i} 1}\left(x_{i}, z_{s_{i}}, \omega\right)=\left(\frac{z_{s_{i}}}{E_{s} I_{s}}\right) \frac{\partial^{2} G_{a}}{\partial x^{2}}\left(x_{i}, \omega\right) \\
\mathbf{A}_{\mathbf{i} 2}\left(x_{i}, z_{s_{i}}, \omega\right)=\left(\frac{z_{s_{i}}}{E_{s} I_{s}}\right) \frac{\partial^{2} G_{-a}}{\partial x^{2}}\left(x_{i}, \omega\right)
\end{array}\right.
$$

This equation can be also rewritten as:

$$
\left[\hat{\varepsilon}_{x x}(\omega)\right]=\left[\begin{array}{ll}
\mathbf{A}_{\mathbf{i} 1}(\omega) & \mathbf{A}_{\mathbf{i} 2}(\omega)
\end{array}\right]\left[\begin{array}{l}
\hat{R}_{1}(\omega) \\
\hat{R}_{2}(\omega)
\end{array}\right]
$$

where $[\hat{\varepsilon}(\omega)]$ represents the vector signals in the frequency domain and has a dimension $n_{f}$ that depends on the number of signals.

From Eq. 6, we obtain:

$$
\left[\begin{array}{l}
\hat{R}_{1}(\omega) \\
\hat{R}_{2}(\omega)
\end{array}\right]=\left[\begin{array}{ll}
\mathbf{B}_{11}(\omega) & \mathbf{B}_{12}(\omega) \\
\mathbf{B}_{\mathbf{2 1}}(\omega) & \mathbf{B}_{\mathbf{2 2}}(\omega)
\end{array}\right]\left[\begin{array}{l}
\mathcal{Q}_{1}(\omega) \\
\mathcal{Q}_{2}(\omega)
\end{array}\right]
$$

where the expression of these 4 components $\mathbf{B}_{\mathbf{i k}}$ of the matrix can be explained from the Eq. (6) as follows:

$$
\left\{\begin{array}{l}
\mathbf{B}_{\mathbf{1 1}}(\omega)=\tilde{F}(\omega)\left[G_{-a}(-a, \omega)+\chi(\omega)\right] \\
\mathbf{B}_{\mathbf{1 2}}(\omega)=\tilde{F}(\omega)\left[-G_{-a}(a, \omega)\right] \\
\mathbf{B}_{\mathbf{2 1}}(\omega)=\tilde{F}(\omega)\left[-G_{a}(-a, \omega)\right] \\
\mathbf{B}_{\mathbf{2 2}}(\omega)=\tilde{F}(\omega)\left[G_{a}(a, \omega)+\chi(\omega)\right]
\end{array}\right.
$$

where $\tilde{F}(\omega)=\frac{E_{s} I_{s}}{\mathcal{K}(\omega) \tilde{D}(\omega)}$. The equivalent loads $\mathcal{Q}_{k}(\omega)$ are calculated on each rail $k$ in the frequency domain (see 
Appendix B) as follows:

$$
\begin{aligned}
\mathcal{Q}_{k}(\omega) & =\frac{\mathcal{K}(\omega)}{v E_{r} I_{r}\left[\left(\frac{\omega}{v}\right)^{4}-\lambda_{r}^{4}\right]} \sum_{j=1}^{K} Q_{k j} \mathrm{e}^{-\mathrm{i} \omega \frac{D_{j}}{v}} \\
& =[\mathbf{C}(\omega)] \mathbf{Q}_{\mathbf{k}}
\end{aligned}
$$

where $\mathbf{Q}_{\mathbf{k}}=\left[Q_{k j}\right]_{j}$ is a column vector of all moving loads on the rail $k$. The matrix $[\mathbf{C}(\omega)]$ has dimensions $\left[n_{f} \times K\right]$ with $n_{f}$ and $K$ represent the length of the vector $\left[\hat{\varepsilon}_{x x}(\omega)\right]$ and the wheel number respectively.

The linear relation between the sleeper strains in the frequency domain and the train loads is obtained by combining Eqs. (10) and (11) as follows:

$$
\left[\hat{\varepsilon}_{x x}(\omega)\right]=[\mathbf{M}(\omega)][\mathbf{Q}]
$$

where

$$
\text { the matrix }
$$

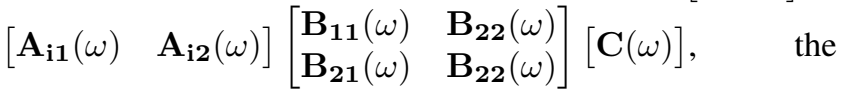
vector $[\mathbf{Q}]=\left[\begin{array}{l}\mathbf{Q}_{1} \\ \mathbf{Q}_{\mathbf{2}}\end{array}\right]$ and this vector does not dependent on the frequency.

Finally, we can use the function mldivide in MATLAB to solve this linear equation to obtain the train loads $[\mathbf{Q}]$ from the Eq. (14). This MATLAB solver has many methods of factorization to solve a system linear equation which depends on the dimension of the matrices. In this case, the matrix $[\mathbf{M}(\omega)]$ is rectangular, thus the method of QR factorization will be used in the mldivide solver.

\subsection{Verification with the analytical signals}

We verify the precision of the "inverse problem" by using the analytical signals generated by the analytical model and perturbation by a white noise. The parameters of the railway track are shown in Table 1. By using the model, the sleeper response subjected to a series of random vertical loads can be calculated in the time and frequency domains from -1000 $(\mathrm{Hz})$ to $1000(\mathrm{~Hz})$. In the case of analytical signals input without noise, the identification method gives us the train load identified with high precision (error $\varepsilon_{r}<4 \times 10^{-4 \%}$ ).

In the next step, we add a random noise to the analytical signals input in time domain to simulate a signal recorded with a passing train. Fig. 2 shows the sleeper strain as a red line and the noise signal as a blue line on the left rail (a) and right rail (b) in a time interval which corresponds to the time for the passing of a train. It shows the case of a large amount of introduced noise in which the level of noise is $25 \%$ corresponding to a strongly noisy signal and the relative error is about $2.6 \%$. By using the inverse problem, the superposition of the train loads introduced and identified is shown in Fig. 3.

Moreover, the Table 2 shows the relative error with different amplitudes of noise in the signal: slightly noisy, noisy, very noisy and strongly noisy. The level of noise $l_{n}$ can be calculated as follows:

$$
l_{n}(\%)=\frac{\sigma_{n}}{\max \left(\varepsilon_{x x}\right)} \times 100
$$

where $\sigma_{n}$ is the standard deviation of the noise imposed on the analytical signal. Thus, according to the Table 2, we can conclude that the identified train loads have been found with a good precision with the analytical signals.
Table 1. Parameters of the railway track (Azoh et al. (2014) and Tran et al. (2018))

\begin{tabular}{l||l|c|l}
\hline Content & Unit & Not. & Value \\
\hline $\begin{array}{l}\text { Young's modulus } \\
\text { of rail }\end{array}$ & $\mathrm{GPa}$ & $E_{r}$ & 210 \\
$\begin{array}{l}\text { Second moment } \\
\text { inertia of rail }\end{array}$ & $\mathrm{m}^{4}$ & $I_{r}$ & $3 \times 10^{-5}$ \\
Rail density & & & \\
Rail section area & $\mathrm{kgm}^{-3}$ & $\rho_{r}$ & 7850 \\
Young's modulus & $\mathrm{GPa}^{2}$ & $S_{r}$ & $7.69 \times 10^{-3}$ \\
of sleeper & & $E_{s}$ & 48 \\
Second moment & $\mathrm{m}^{4}$ & $I_{s}$ & $1.69 \times 10^{-4}$ \\
inertia of sleeper & & & \\
Density of sleeper & $\mathrm{kgm}^{-3}$ & $\rho_{s}$ & 2658 \\
Sleeper section area & $\mathrm{m}^{2}$ & $S_{s}$ & $47.7 \times 10^{-3}$ \\
Length of sleeper & $\mathrm{m}$ & $2 L$ & 2.41 \\
Track gauge & $\mathrm{m}$ & $2 a$ & 1.435 \\
Stiffness of ballast & $\mathrm{MNm}^{-1}$ & $k_{f}$ & 240 \\
Damping coefficient & $\mathrm{kNsm}^{-1}$ & $\zeta_{f}$ & 58.8 \\
of ballast & & & \\
Stiffness of rail pad & $\mathrm{MNm}^{-1}$ & $k_{r p}$ & 192 \\
Damping coefficient & $\mathrm{MNsm}$ & $\zeta_{r p}$ & 1.97 \\
of rail pad & & & \\
Train speed & $\mathrm{ms}^{-1}$ & $v$ & 50 \\
Pre-stress & $\mathrm{kN}^{-1}$ & $T$ & 300 \\
Sleeper spacing & $\mathrm{m}$ & $l$ & 0.6 \\
\hline
\end{tabular}

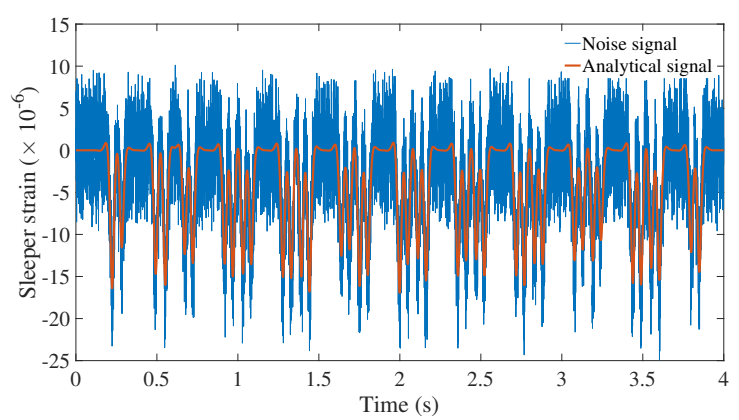

(a) Left rail

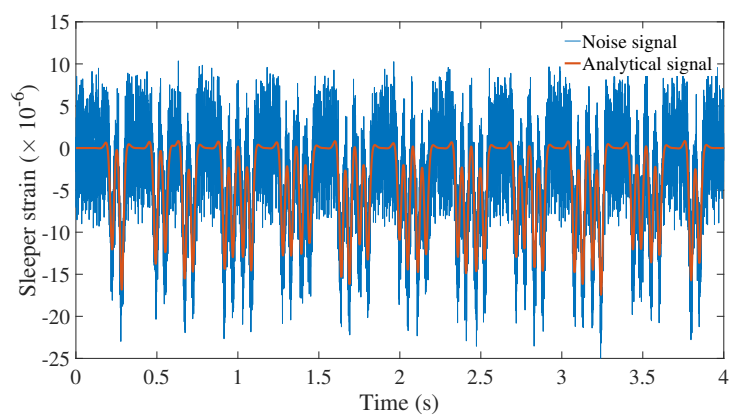

(b) Right rail

Figure 2. Analytical and noise signals on the left rail (a) and right rail (b)

\section{Experimental validations}

In this section, we built a verification tests with an impact hammer and a passing train. The sleeper is integrated by the strain gauges KYOWA KC-70-120-A1-11 as shown in the Fig. 4. In this experiment, 7 gauges situated between the two 


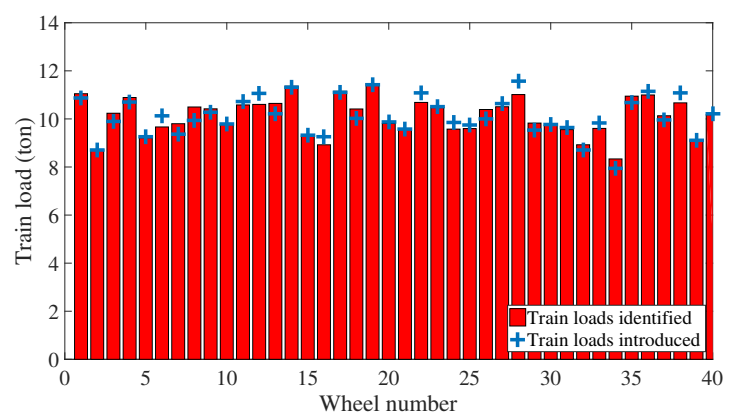

(a) Left rail

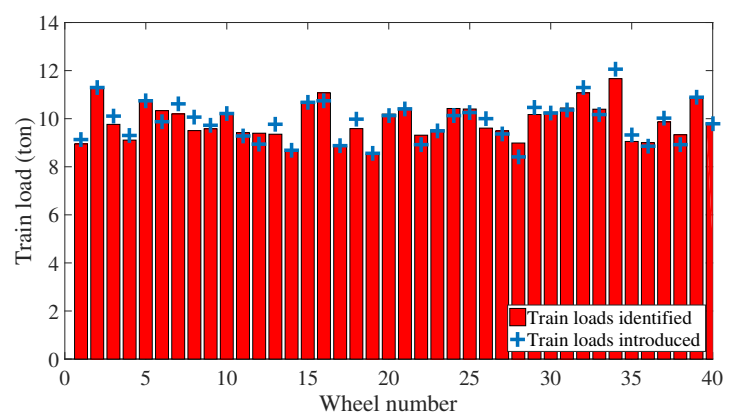

(b) Right rail

Figure 3. Comparison of the introduced (blue) and identified train loads (red) on the left rail (a) and right rail (b)

Table 2. Relative error between the train loads introduced and identified for different noise levels

\begin{tabular}{l||l|l|l}
\hline Level of noise $l_{n}(\%)$ & Left rail & Right rail & Average \\
\hline $2 \%$ (Slightly noisy) & $0.281 \%$ & $0.285 \%$ & $0.283 \%$ \\
$4.5 \%$ (Noisy) & $0.585 \%$ & $0.597 \%$ & $0.592 \%$ \\
$12 \%$ (Very noisy) & $1.186 \%$ & $1.159 \%$ & $1.172 \%$ \\
$25 \%$ (Strongly noisy) & $2.541 \%$ & $2.634 \%$ & $2.588 \%$ \\
\hline
\end{tabular}

rails at equal distance. The $8^{\text {th }}$ gauge is on the opposite face and in the middle of the crossbar (identical position to the $4^{\text {th }}$ in the length sleeper direction - see Fig. 4).

\subsection{Identify a force generated by an impact hammer}

An impact hammer PCB Piezotronic 086D50 is used to excite a vertical force on a sleeper at different positions (see Fig. 5). During the test, the hammer gives a signal which allows us to calculate the force generated. The sleeper strains are recorded with the help of a software developed by the Laboratoire Navier, École des Ponts ParisTech. By using the method of identification of the train load, the force generated on the sleeper via the impact hammer will be identified from the signals recorded by the strain gauges.

Fig. 6 shows an example of the sleeper strains recorded by the strain gauges during one excitation. Here, we show the sleeper strain of the strain gauge 4 (continuous-blue line) and strain gauge 2 (dot-green line). In the same figure, the red line presents a force identified. We note that the shape of the force identified in the Fig. 6 in time domain is not similar the Dirac's distribution. This is normal because the free movements of the sleeper are recorded in the signals. The value of the force can be obtained at the first peak of the

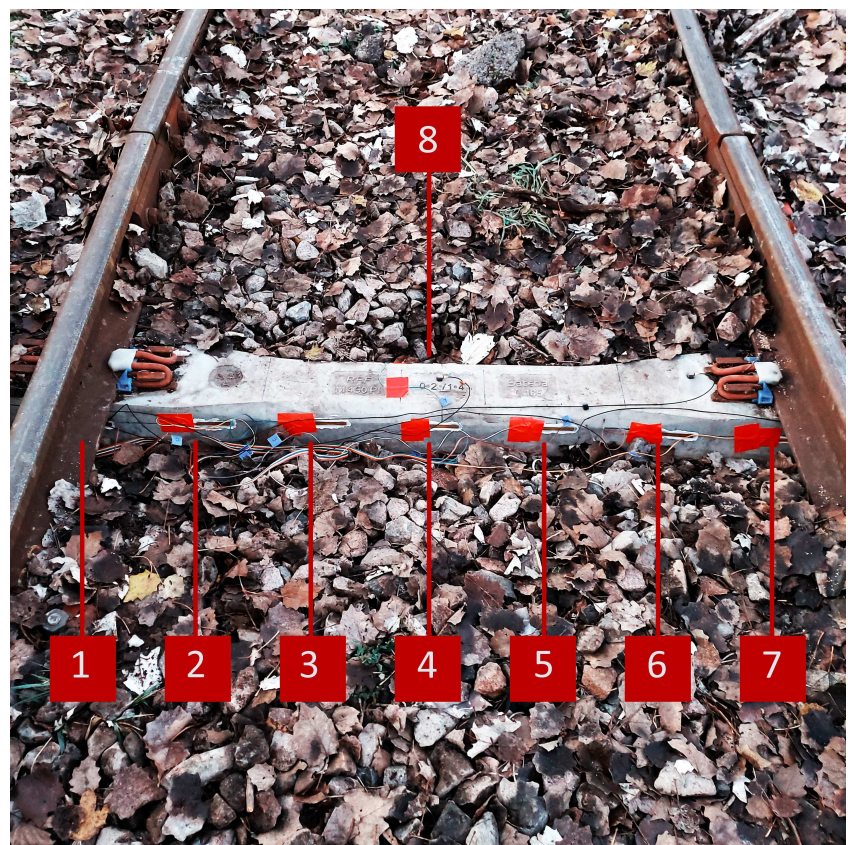

Figure 4. Sleeper integrated by the strain gauges in the validation test

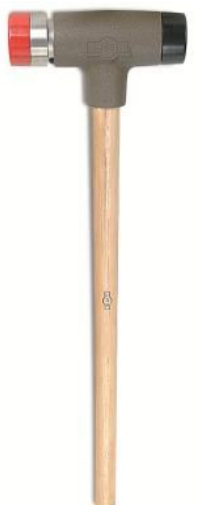

Figure 5. Impact hammer PCB 086D50

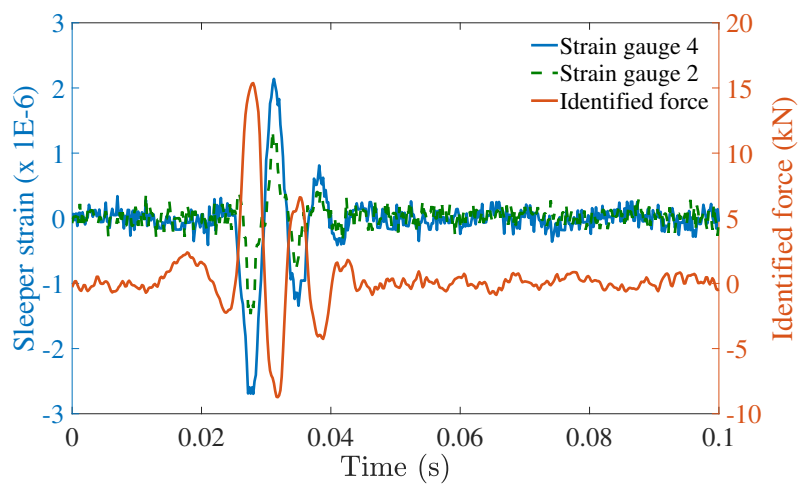

Figure 6. Sleeper strains recorded during an excitation by using the impact hammer and identified force

signals. In addition, the sleeper train and the force identified are in the opposite phase because the area where the gauges are located is in compression.

The Table 3 shows the comparison of the force identified and generated at 3 different positions of the excitation (at the center, nearly the rail position and at the end of sleeper). 
Table 3. Comparison of the force identified and generated by the hammer

\begin{tabular}{l||c|c|c}
\hline & Test 1 & Test 2 & Test 3 \\
\hline Force generated & $14.93 \mathrm{kN}$ & $16.57 \mathrm{kN}$ & $14.81 \mathrm{kN}$ \\
Force identified & $15.28 \mathrm{kN}$ & $16.32 \mathrm{kN}$ & $14.54 \mathrm{kN}$ \\
Relative error & $2.34 \%$ & $1.534 \%$ & $1.82 \%$ \\
\hline
\end{tabular}

We see that this method allows us to identify the forces with good precision (the relative error is less than $2.5 \%$ ). The error of the method could be explained by noises of measurements. In addition, the position of application of the force is not sufficiently precise. Therefore, we can conclude that the method and the parameters of the railway track are valid.

\subsection{Identify train loads with a passing train}

A second test has been realized with a passing train. In this experiment, the train includes 4 wagons which are loaded with sleepers manufactured in the SATEBA factory (see Fig. 7) and it passes on the integrated sleeper. The characteristics of each wagon are identified, especially the mass of wagons, the bogie length and the load (number of sleepers) on each wagon.

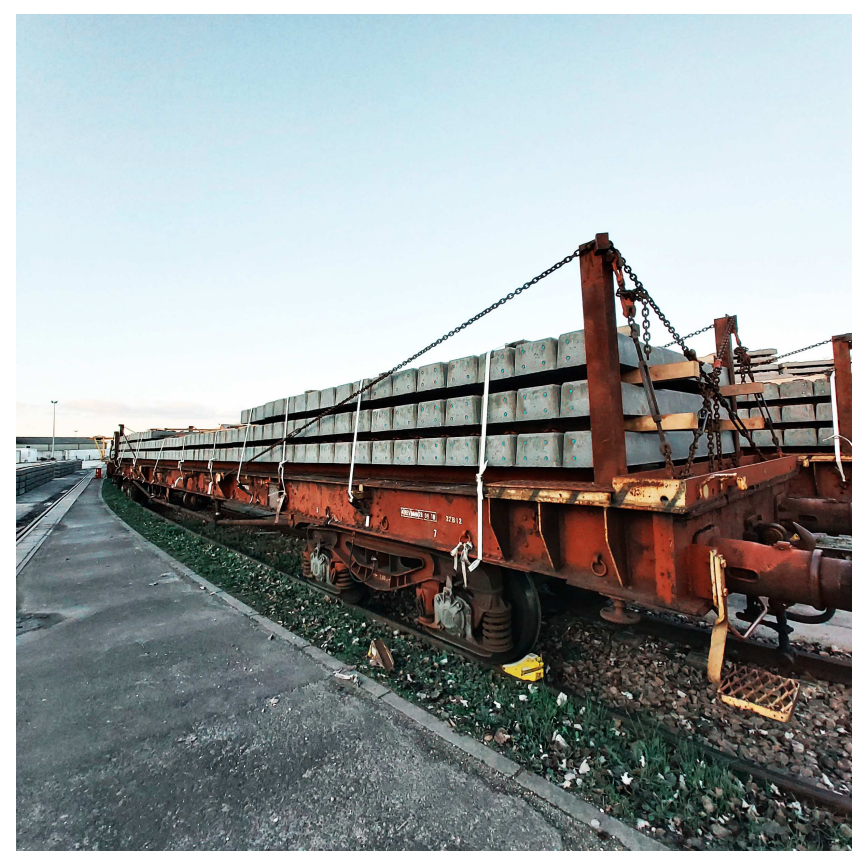

Figure 7. Wagons loaded by the sleepers in the validation test

The sleeper strains recorded by the 8 gauges during the passing train are shown in the Fig. 8. There are 20 peaks which correspond to the 20 wheels of the train on each rail (1 locomotive and 4 wagons). Because the first wheel of the locomotive was placed very close to the sleeper, therefore it was deformed before the start of the measurement. For this reason, we only identify wagon loads. The first wheel of the first wagon is counted from the $5^{\text {th }}$ peak. We note that when the wagons pass, the signals recorded are very clear.

Fig. 9 presents the loads identified of each wheel on two rails by using the method of identification of the train loads. Besides, the Table 4 presents the identified

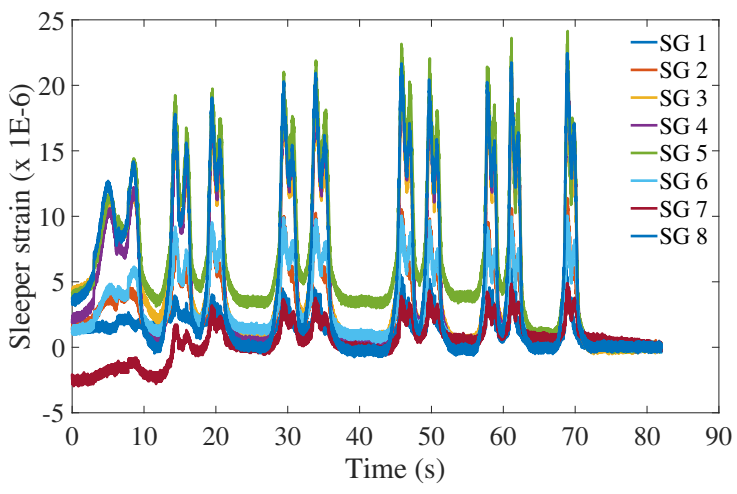

Figure 8. Signals recorded during the passing train in the validation test (SG: Strain gauge)

masses of 4 wagons. By comparing these masses with the reference masses of each wagon, we note a maximum relative difference is $5.5 \%$. The errors could be explained by the condition of the foundation, the noises integrated in the measurement or a small variation on the reference mass. However, these relative errors remain small enough to be able to conclude that this method that we have presented is validated.

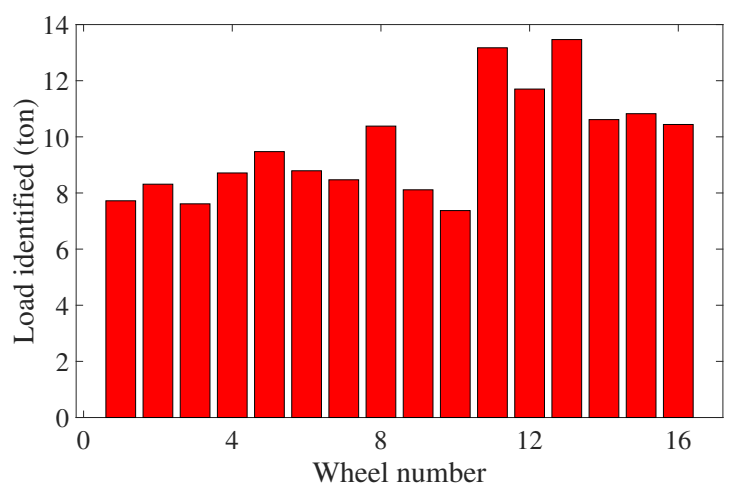

(a) Loads identified at the rail 1

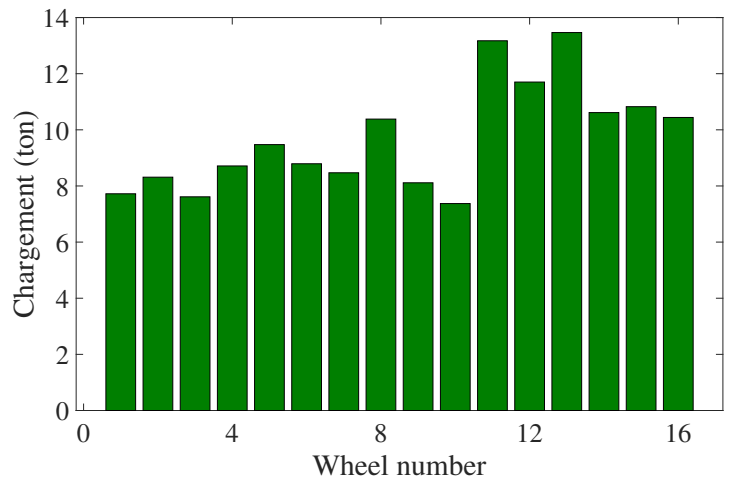

(b) Loads identified at the rail 2

Figure 9. Train loads identified in the validation test 
Table 4. Comparison of identified loads of a train

\begin{tabular}{l||c|c|c}
\hline $\begin{array}{l}\text { Wagon's } \\
\text { number }\end{array}$ & $\begin{array}{c}\text { Total } \\
\text { mass }\end{array}$ & $\begin{array}{c}\text { Mass } \\
\text { identified }\end{array}$ & $\begin{array}{c}\text { Relative } \\
\text { error }\end{array}$ \\
\hline $1^{\text {st }}$ wagon & 65.95 ton & 62.37 ton & $5.43 \%$ \\
$2^{\text {nd }}$ wagon & 75.33 ton & 73.36 ton & $2.61 \%$ \\
$3^{\text {rd }}$ wagon & 66.95 ton & 68.95 ton & $2.98 \%$ \\
$4^{\text {th }}$ wagon & 72.33 ton & 76.29 ton & $5.48 \%$ \\
\hline
\end{tabular}

\section{Application with the measurements in-situ}

A "Smart Sleeper" developed by Sateba with 6 Fiber Bragg Grating sensors (FBG) integrated in the longitudinal direction allow us to obtain the sleeper strain at the passing train as shown in the Fig. 10. These sensors are situated at the two rail seats and at the middle of the sleeper. Mechanical tests have been carried out in the laboratory by the laboratoire Navier at École des Ponts ParisTech and SATEBA to validate the quality of the FBG sensors. The signals recorded in-situ by these sensors are transformed in the frequency domain with the help of the Fourier's transform. The sampling frequency of the FBG sensors is $1000 \mathrm{~Hz}$. The maximum strain measurable is $5000 \mu \mathrm{def}$.

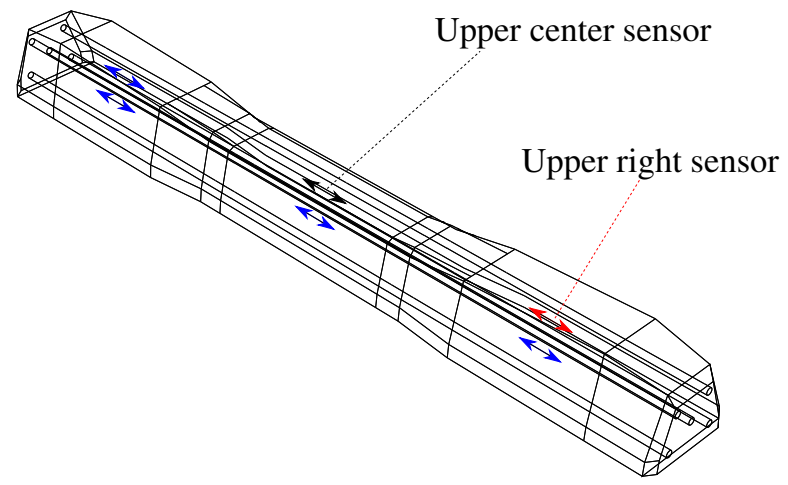

Figure 10. "Smart sleeper": upper right sensor (red) and upper center sensor (black)

In this section, we apply the method of identification of the train loads to the measurement in-situ. The sleeper strains have been recorded in-situ with the help of the "Smart Sleeper" at Creil, France, on the $6^{\text {th }}$ May 2017. Fig. 11 shows the signal recorded by the "Smart Sleeper" during the passing train (green and red lines). This train contains 1 locomotive and 9 wagons. The locomotive and each wagon have two bogies, each bogie has 2 axles. Consequently, the locomotive and each wagon have 4 wheels on each rail. The green and red line represent the measured strain on the upper left (a) and right (b) sensor respectively. By using the identified train loads, we rebuild a sleeper strain with the help of the analytical model. In the Fig. 11, the rebuilt responses are represented by the black lines. The black lines show the sleeper strain in the perfect condition (without noise). This is the reason why the rebuilt signals are smooth and their amplitudes are smaller than the measurements (green and red lines).

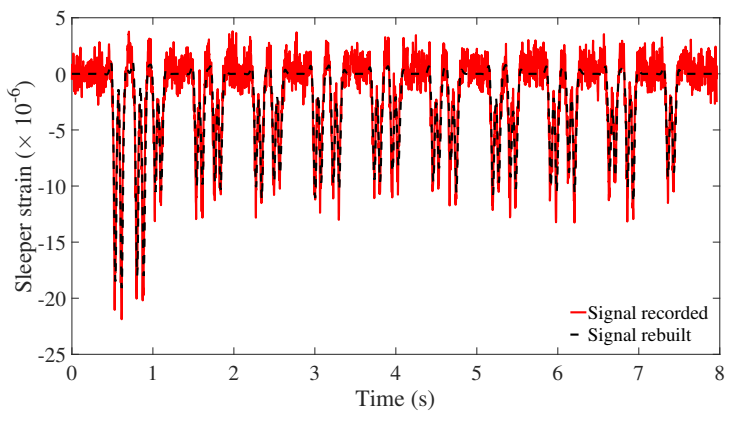

(a) Upper left sensor

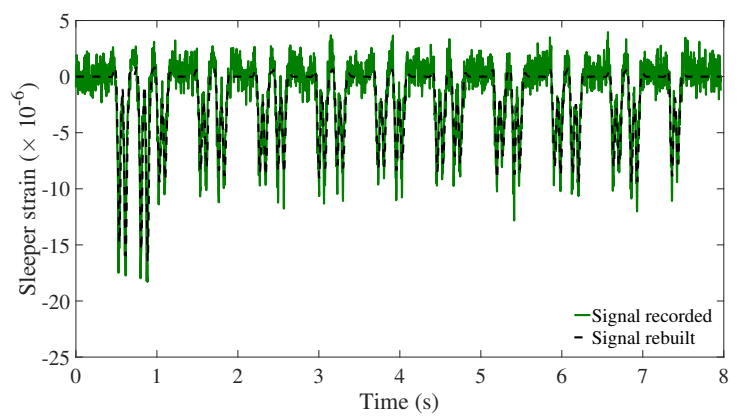

(b) Upper right sensor

Figure 11. Sleeper response on the left rail (a) and right rail (b)

The train speed calculated is: $36.5\left(\mathrm{~ms}^{-1}\right)$. The other track parameters are the same as in the Table 1. Fig. 12 presents the vertical identified train loads.

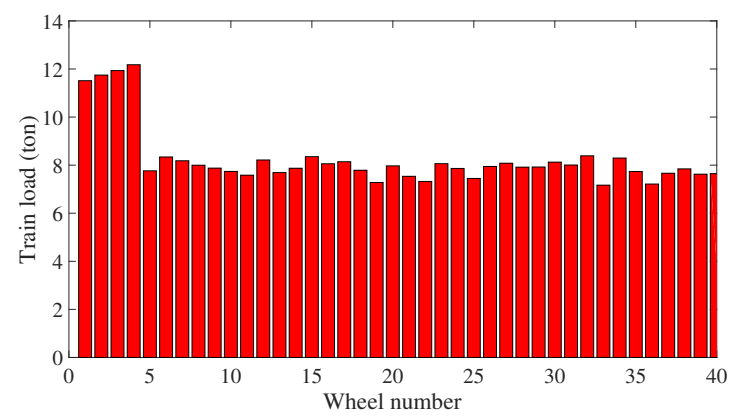

(a) Left rail

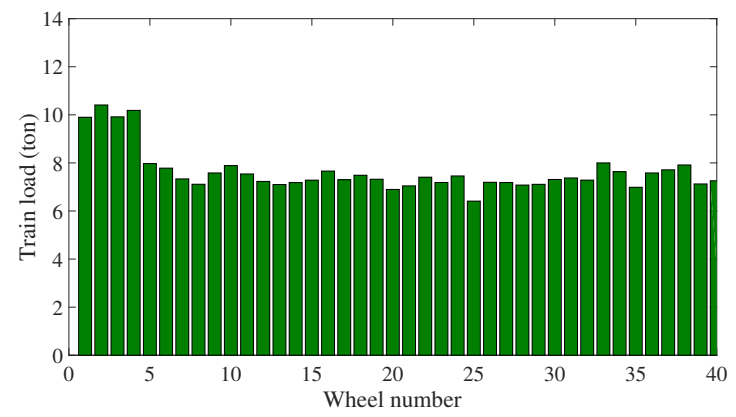

(b) Right rail

Figure 12. Train loads identified by using the measurements on the left rail (a) and right rail (b)

The Table 5 shows the mass of the locomotive and the wagons by calculating the sum of four train loads on the two rails. The value of the first four loads corresponding to the locomotive of the train on the two rails are bigger than 
Table 5. The weight of the locomotive and each wagon

\begin{tabular}{l||c}
\hline Wagon number & Mass \\
\hline Locomotive & 87.78 ton \\
$1^{\text {st }}$ wagon & 62.48 ton \\
$2^{\text {nd }}$ wagon & 61.65 ton \\
$3^{\text {rd }}$ wagon & 61.20 ton \\
$4^{\text {th }}$ wagon & 60.18 ton \\
$5^{\text {th }}$ wagon & 59.88 ton \\
$6^{\text {th }}$ wagon & 59.25 ton \\
$7^{\text {th }}$ wagon & 61.52 ton \\
$8^{\text {th }}$ wagon & 60.62 ton \\
$9^{\text {th }}$ wagon & 60.79 ton \\
\hline
\end{tabular}

the rest, and explains that the locomotive is heavier than the wagons. We see this phenomenon also on the sleeper response in the Fig. 11. By comparing with the document of SNCF ${ }^{\ddagger}$ published by Redoutey (2016), the weight of the locomotive identified in this example are closed to the value of reference $(87.78 \mathrm{kN}$ to compare with $90 \mathrm{kN})$. Moreover, the identified mass is almost the same for the 9 wagons.

In addition, Fig. 11 shows that the sleeper strains at the two rail seats are not equal in value (the sleeper strain at the right rail is higher than for the left rail). By identifying the train loads, this phenomenon is demonstrated by the different vertical train loads on the two rails. This could be explained by the unbalanced of rolling stock.

We note that the behavior of the ballast-foundation is one of factor which can relate to the difference of the sleeper strains at the two rail-seat positions. For example, with a non-homogeneous foundation, the train loads could be identified with error. In this study, we limit the homogeneous foundation (consolidated foundation) of the railway track and the factor of consolidation of foundation will be an object of the future work.

\section{Conclusions}

Based on the analytical dynamic model of the railway sleepers, the "inverse problem" has been developed to identify the vertical train loads. In the frequency domain, the sleeper strain and the vertical train loads can be linked by a linear relation. The numerical application gives a good precision with the noisy analytical signal. In addition, the experimental validations have been realized by using an impact hammer and a passing train. The forces identified in the validation tests agree well to the forces generated. An application with the measurements in-situ shows that the train loads identified have different values for each wheel of the train. Thus, this technique can detect the imperfection of the wheel-rail contact when the train load on one rail is much higher than the other. Moreover, the application of the measurements in-situ shows the mass of wagons and the mass of the locomotive is heavier than the wagons. The identified mass of the locomotive corresponds well to the value of reference. Besides, a verification test is in process. By quantifying wheel loads, this work assists in the management of wheel health and improve maintenance of the rolling stock. In addition, by considering the homogeneous foundation, this application shows the unbalanced of rolling stock which is found in the measurements in-situ at two rail seats. In future works, the model should be developed to identify the vertical train loads in the case of nonhomogeneous foundation.

\section{A Green's function}

By using the Fourier transform and combining equations (1), (2), this system dynamics equation can be rewritten in the frequency domain :

$$
\left\{\begin{array}{c}
\frac{\partial^{4} \hat{w}_{s}}{\partial x^{4}}(x, \omega)-\left(\frac{T}{E_{s} I_{s}}\right) \frac{\partial^{2} \hat{w}_{s}}{\partial x^{2}}(x, \omega) \\
-\left(\frac{\rho_{s} S_{s} \omega^{2}-k_{b}}{E_{s} I_{s}}\right) \hat{w}_{s}(x, \omega) \\
=-\frac{\hat{R}_{1}}{E_{s} I_{s}} \delta(x-a)-\frac{\hat{R}_{2}}{E_{s} I_{s}} \delta(x+a) \\
\frac{\partial^{2} \hat{w}_{s}}{\partial x^{2}}(-L, t)=\frac{\partial^{2} \hat{w}_{s}}{\partial x^{2}}(L, t)=0 \\
\frac{\partial^{3} \hat{w}_{s}}{\partial x^{3}}(-L, t)=\frac{\partial^{3} \hat{w}_{s}}{\partial x^{3}}(L, t)=0
\end{array}\right.
$$

where the symbol $\frac{\partial}{\partial x}$ stands for the partial derivative with regard to $x$ and $k_{b}=k_{f}+\mathrm{i} \omega \zeta_{f}$ is the foundation dynamic stiffness. The Green's function $G_{a}(x, \omega)$ of Eq. (16) is defined by :

$$
\begin{aligned}
& \frac{\partial^{4} G_{a}}{\partial x^{4}}(x, \omega)-\alpha_{s}^{2} \frac{\partial^{2} G_{a}}{\partial x^{2}}(x, \omega)-\lambda_{s}^{4} G_{a}(x, \omega) \\
& =\delta(x-a)
\end{aligned}
$$

where $\alpha_{s}=\sqrt{\frac{T}{E_{s} I_{s}}}$ and $\lambda_{s}=\sqrt[4]{\frac{\rho_{s} S_{s} \omega^{2}-k_{b}}{E_{s} I_{s}}}$ are two constants defined in the Eq. (16a). This is a $4^{\text {th }}$ order linear differential equation and its Green's function Zauderer (1989) can be written as follows:

$$
G_{a}(x, \omega)= \begin{cases}\sum_{i=1}^{4} A_{i} \mathrm{e}^{\lambda_{i} x} & \forall x \in[-L, a] \\ \sum_{i=1}^{4} B_{i} \mathrm{e}^{\lambda_{i} x} & \forall x \in[a, L]\end{cases}
$$

where $A_{i}, B_{i}$, and $\lambda_{i}$ (with $1 \leq i \leq 4$ ) are parameters to be determined and $\lambda_{i}$ are the 4 complex roots of the characteristic equation:

$$
\mathbb{P}(\lambda)=\lambda^{4}-\alpha_{s}^{2} \lambda^{2}-\lambda_{s}^{4}
$$

By using the boundary conditions of the free-free beam (continuity condition for displacement, slope and moments, discontinuity of magnitude one at the point force), we can obtain analytical expressions for $A_{i}, B_{i}$.

\section{B Periodically supported beam model}

The periodically supported beam is shown in Figure 13. When the rails are modeled by periodically supported beams

¥Société Nationale des Chemins de Fer 


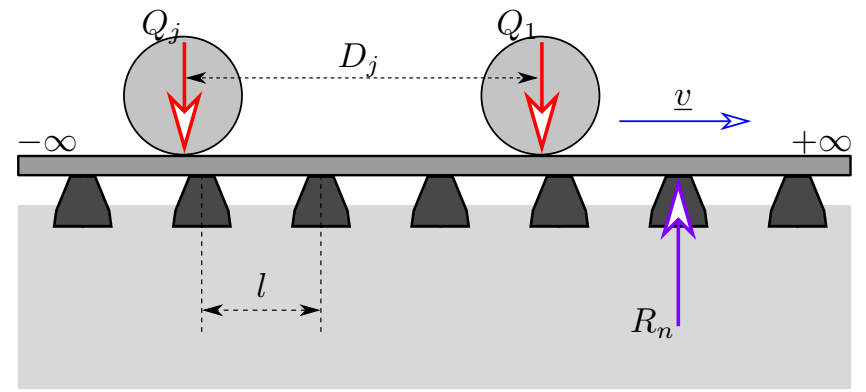

Figure 13. Periodically supported beam subjected to moving loads

Hoang et al. (2017), the forces $R_{k}$ on each rail $k$ in the frequency domain can be calculated as follows:

$$
\hat{R}_{k}(\omega)=\mathcal{K} \hat{w}_{r_{k}}(\omega)+\mathcal{Q}_{k}(\omega)
$$

where $\mathcal{K}$ is the equivalent stiffness, $\mathcal{Q}_{k}$ are the equivalent loads of the two rails which are determined. In another way, a rail pad can be modelled as a system of springer-damper which has the stiffness $k_{r p}$ and the damping coefficient $\zeta_{r p}$ respectively. Let $w_{r p}(t)$ be the displacement of the rail pad in the time domain, the reaction force applied on the sleeper at the rail $k$ can be calculated through the rail pad as follows:

$$
R_{k}(t)=-\left[k_{r p} w_{r p}(t)+\zeta_{r p} \frac{\partial w_{r p}(t)}{\partial t}\right]
$$

Moreover, the rail pad displacement can be deduced by calculating the difference of the rail displacement $w_{r_{k}}(t)$ and sleeper displacement at the rail position $w_{s_{k}}(t)$ :

$$
w_{r p}(t)=w_{r_{k}}(t)-w_{s_{k}}(t)
$$

By substituting Eq. (22) into Eq. (21) and using the Fourier's transform, the reaction force $\hat{R}_{k}$ applied on the sleeper can be expressed in the frequency domain as follows:

$$
\hat{R}_{k}(\omega)=-k_{p}\left(\hat{w}_{r_{k}}(\omega)-\hat{w}_{s_{k}}(\omega)\right)
$$

where $k_{p}=k_{r p}+\mathrm{i} \omega \zeta_{r p}$ is the dynamic stiffness of the rail pad, $\hat{w}_{r_{k}}$ and $\hat{w}_{s_{k}}$ are respectively the displacements of the rail $k$ and sleeper in the frequency domain. Finally, by combining Eq. (20) into Eq. (23), we obtain:

$$
\hat{R}_{k}(\omega)=\frac{k_{p} \mathcal{K}}{k_{p}+\mathcal{K}} \hat{w}_{s_{k}}(\omega)+\frac{k_{p}}{k_{p}+\mathcal{K}} \mathcal{Q}_{k}(\omega)
$$

\section{Acknowledgements}

This work has been developed in the context of a partnership between Sateba (Consolis Group) and Ecole des Ponts ParisTech. The authors would like to thank the personnel of Sateba for their support.

\section{References}

Arab AA, Badie SS and Manzari MT (2011) A methodological approach for finite element modeling of pretensioned concrete members at the release of pretensioning. Engineering Structures 33(6): 1918-1929. DOI:10.1016/j.engstruct.2011. 02.028 .
Azoh TS, Nzie W, Djeumako B and Fotsing BS (2014) Modeling of train track vibrations for maintenance perspectives: application. European Scientific Journal 10(21): 260-275.

Buggy SJ, James SW, Staines S, Carroll R, Kitson P, Farrington D, Drewett L, Jaiswal J and Tatam RP (2016) Railway track component condition monitoring using optical fibre bragg grating sensors. Measurement Science and Technology 27(5): 055201.

Da Costa Marques Pimentel RM, Barbosa MCB, Costa NMS, Ribeiro DRF, de Almeida Ferreira LA, Araujo FMM and Calcada RAB (2008) Hybrid fiber-optic/electrical measurement system for characterization of railway traffic and its effects on a short span bridge. IEEE Sensors Journal 8(7): 1243-1249. DOI:10.1109/JSEN.2008.926519.

Ding H, Chen LQ and Yang SP (2012) Convergence of Galerkin truncation for dynamic response of finite beams on nonlinear foundations under a moving load. Journal of Sound and Vibration 331(10): 2426-2442. DOI:10.1016/j.jsv.2011.12. 036.

Filograno ML, Corredera Guillen P, Rodriguez-Barrios A, MartinLopez S, Rodriguez-Plaza M, Andres-Alguacil and GonzalezHerraez M (2012) Real-time monitoring of railway traffic using fiber bragg grating sensors. IEEE Sensors Journal 12(1): 8592. DOI:10.1109/JSEN.2011.2135848.

Ho SL, Lee KK, Lee KY, Tam HY, Chung WH, Liu SY, Yip CM and Ho TK (2006) A comprehensive condition monitoring of modern railway. In: 2006 IET International Conference On Railway Condition Monitoring. pp. 125-129.

Hoang T, Duhamel D, Foret G, Yin HP, Joyez P and Caby R (2017) Calculation of force distribution for a periodically supported beam subjected to moving loads. Journal of Sound and Vibration 388: 327-338. DOI:10.1016/j.jsv.2016.10.031.

Kumaran G, Menon D and Krishnan Nair K (2003) Dynamic studies of railtrack sleepers in a track structure system. Journal of Sound and Vibration 268(3): 485-501. DOI:10.1016/ S0022-460X(02)01581-X.

Loaëc A and Petit C (2014) Measurement of bending moments in concrete sleepers laid on ballast tracks. In: Transport Research Arena (TRA) $5^{\text {th }}$ Conference: Transport Solutions from Research to Deployment.

Mead D (1970) Free wave propagation in periodically supported, infinite beams. Journal of Sound and Vibration 11(2): 181197. DOI:10.1016/S0022-460X(70)80062-1.

Mead D (1996) Wave propagation in continuous periodic structures: Research contributions from Southampton, 1964 - 1995. Journal of Sound and Vibration 190(3): 495-524. DOI:10. 1006/jsvi.1996.0076.

Nguyen VH and Duhamel D (2006) Finite element procedures for nonlinear structures in moving coordinates. Part 1: Infinite bar under moving axial loads. Computers \& Structures 84(21): 1368-1380. DOI:10.1016/j.compstruc.2006.02.018.

Nguyen VH and Duhamel D (2008) Finite element procedures for nonlinear structures in moving coordinates. Part II: Infinite beam under moving harmonic loads. Computers \& Structures 86(21): 2056-2063. DOI:10.1016/j.compstruc.2008.04.010.

Qian Y, Dersch MS, Gao Z and Edwards JR (2019) Railroad infrastructure 4.0: Development and application of an automatic ballast support condition assessment system. Transportation Geotechnics DOI:10.1016/j.trgeo.2019.01.002. 
Redoutey D (2016) Le matériel moteur de la SNCF en 2016. La vie du Rail. ISBN 978-2-915034-65-3.

Sheng X, Jones C and Thompson D (2005) Responses of infinite periodic structures to moving or stationary harmonic loads. Journal of Sound and Vibration 282(1): 125-149. DOI:10. 1016/j.jsv.2004.02.050.

Tam HY, Ho SL, Lee KY, Haber T, Graver T and Mendez A (2007) Utilization of fiber optic bragg grating sensing systems for health monitoring in railway applications. In: 6th International Workshop on Structural Health Monitoring. pp. 1824-1833.

Tran LH, Hoang T, Foret G, Duhamel D, Messad S and Loaëc A (2018) A fast analytic method to calculate the dynamic response of railways sleepers. Journal of Vibration and Acoustics 141(1): 1368-1380. DOI:10.1115/1.4040392.

Wei C, Lai C, Liu SY, Chung WH, Ho TK, Tam HY, Ho SL, MacCusker A, Kam J and Lee KY (2010) A fiber bragg grating sensor system for train axle counting. IEEE Sensors Journal 10(12): 1905-1912. DOI:10.1109/JSEN.2010.2049199.

Wei C, Xin Q, Chung WH, Liu SY, Tam HY and Ho SL (2012) Real-time train wheel condition monitoring by fiber bragg grating sensors. International Journal of Distributed Sensor Networks 8(1): 409048. DOI:10.1155/2012/409048.

Yazdizadeh Z, Marzouk H and Hadianfard MA (2017) Monitoring of concrete shrinkage and creep using Fiber Bragg Grating sensors. Construction and Building Materials 137: 505-512. DOI:10.1016/J.CONBUILDMAT.2017.01.084.

Zauderer E (1989) Partial differential equations of applied mathematics. John Wiley Sons. ISBN 0-471-87517-1. 